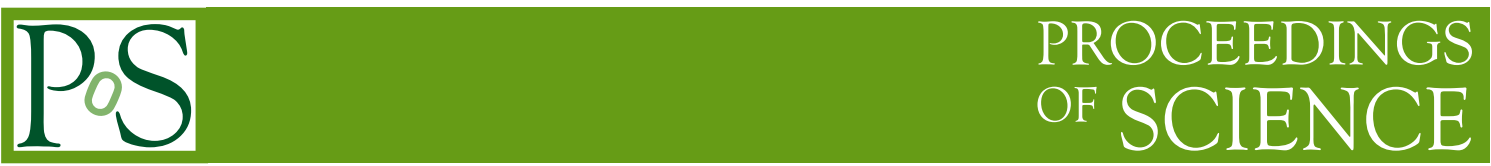

\title{
Black hole spin estimation
}

\section{Odele Straub *}

N. Copernicus Astronomical Center

E-mail: odele@camk.edu.pl

\begin{abstract}
Astrophysical black holes are defined by two quantities: their mass $M$ and their spin $a$. Whereas black hole masses in X-ray binaries can be determined directly through dynamical optical studies of the companion star, spin estimation is only possible indirectly by interpreting the diversity of physical processes that govern electromagnetic radiation emitted by the accretion disc surrounding a black hole. Two fundamentally different techniques to measure the black hole spin of microquasars from the observed X-ray spectra are now being elaborated and refined. One approach is fitting the continuum emission with a slim disc model for an advective accretion flow. The other approach is comparing the high frequency twin-peak quasi-periodic oscillations to those performed by a non-slender accretion torus. In both models the Kerr metric provides the background geometry. The aim is to independently measure black hole spins with different methods. Here I report on spin estimations of three microquasars, GRO J1655-40, XTE J1550-564 and GRS $1915+105$.
\end{abstract}

VII Microquasar Workshop: Microquasars and Beyond September 1 - 5, 2008

Foca, Izmir, Turkey

*The two discussed projects have been carried out in collaboration with M. Abramowicz, M. Bursa, W. Kluźniak, R. Narayan, O. Sạdowski, R. Shafee and E. Šrámková. 


\section{Contents}

1. Introduction 2

2. Spectral fitting of stationary slim discs $\quad 2$

2.1 Spin estimation 3

2.2 Data selection 3

3. HF QPOs 3

3.1 Epicyclic oscillations of non-slender tori 4

4. Comparison 5

\section{Introduction}

When we study the black hole spin, we examine distant electromagnetic radiation emitted by matter that is being accreted into the black hole $(\mathrm{BH})$. We have reason to assume that the majority of astrophysical black holes have due to the way they are formed a non-zero angular momentum, $J$ (dimensionless, $a=J c /\left(G M^{2}\right)$ ). While mass provides a scale, the spin changes the space-time in the vicinity of a $\mathrm{BH}$. Observed radiation close to the $\mathrm{BH}$ therefore contains exact information about the BH spin. However, this information is concealed by the complex accretion physics. Several important aspects, in particular viscosity and radiative transfer, remain not sufficiently known such that it is not yet possible to accurately calculate properties of the radiation emitted by accretion flows. Consequently, one either has to work with simplified models that require specific conditions or sidestep to a model that is not affected by radiative transfer. This article briefly reviews two fundamentally different techniques to estimate the BH spin from the observed X-ray spectra of microquasars.

\section{Spectral fitting of stationary slim discs}

The basic idea is to fit the modelled spectrum of a stationary slim disc around a Kerr black hole to the observed thermal X-ray spectrum of a microquasar, having $\mathrm{BH}$ spin and mass accretion rate as fit parameters. The crucial difference to the standard thin disc model is that slim accretion discs have a scale height $H / R<1$ and are cooled by radiation and advection. The mass accretion rate $\dot{M} \geq \dot{M}_{E d d}$ gives rise to a transsonic accretion flow that continues inside the innermost stable circular orbit (the critical radius is given by the location of the sonic point). Thus, heat is lost through the inner disc radius, and especially at higher accretion rates, the efficiency goes down. Slim accretion disc models take advantage of simplification due to vertical integration (as used for thin discs), apply, however, at the same time the correct thick disc approach to the transsonic part 
of the flow. In a stationary ${ }^{1}$, hydrodynamical set-up (no magnetic fields present) with radiative cooling and $\alpha$-viscosity prescription, the five disc equations (mass, angular momentum and energy conservation as well as radial momentum and vertical equilibrium) can be reduced to two differential equations for the radial velocity of the flow, $v_{r}$ and the temperature, $T_{0}$. These two profiles vary for different values of $\alpha, M_{B H}, \dot{M}$ and $a$. With the (multicolour) blackbody assumption i.e., the disc radiates at each radius like a blackbody, the flux profile, $F_{\text {disc }}$, of a slim accretion disc can then be constructed.

\subsection{Spin estimation}

Black holes have no solid surface, instead they have an event horizon. For maximally rotating BHs it can get as small as half a Schwarzschild radius. In general relativity there exists a last (innermost) stable circular orbit, $R_{I S C O}$ beyond which a particle heads in free-fall towards the event horizon and inside the $\mathrm{BH}$. This radius depends on the $\mathrm{BH}$ spin and marks the inner edge of an accretion disc.

In principle, $R_{I S C O}$ can be found like the radius $R_{\star}$ of a star from the luminosity

$$
L_{\star}=4 \pi D^{2} F_{\star}(R, i)=4 \pi R_{\star}^{2} \sigma T^{4} .
$$

Once distance, $D$, inclination and $i$ are known the observed flux $F_{\star}$ leads (via BB assumption) to temperature and radius. To get the location of the marginally stable radius $R_{I S C O}$ from the observed flux $F_{\text {disc }}(R, i)$ one needs the disc luminosity for a mass accretion rate $\dot{M}$,

$$
L_{d i s c}=\eta \dot{M} c^{2},
$$

where $\eta$ is the efficiency, and creates a model spectrum assuming the disc is in a thermal state.

Thermal spectra of X-ray binaries GRO J1655-40, GRS 1915+105 and 4U 1543-47, were collected e.g., by RXTE and fitted with the standard thin disc model by [10]. Spin estimation based on the mentioned slim disc model is currently being carried out shall be presented in a separate article.

\subsection{Data selection}

Since the blackbody assumption is only valid for disc dominated spectra, a rigorous selection of data (cf. [8]) from thermal microquasar states is of utmost importance. The purpose of this data selection is to minimise the very uncertain effects of comptonisation because we have only the crudest knowledge of coronal comptonising i.e., the geometry and physical conditions of the electron scattering region. Spectra of other disc states with state-of-the-art comptonisation models were carried out by [5] and [6].

\section{HF QPOs}

Many known low mass X-ray binaries show quasi-periodic variability in their X-ray flux, that are seen in the Fourier power density spectrum as frequency peaks. High frequency QPOs are

\footnotetext{
${ }^{1}$ Slim discs are thermally and viscously unstable and show at a given radius a characteristic S-shaped equilibrium curve on an accretion rate versus surface density plane.
} 

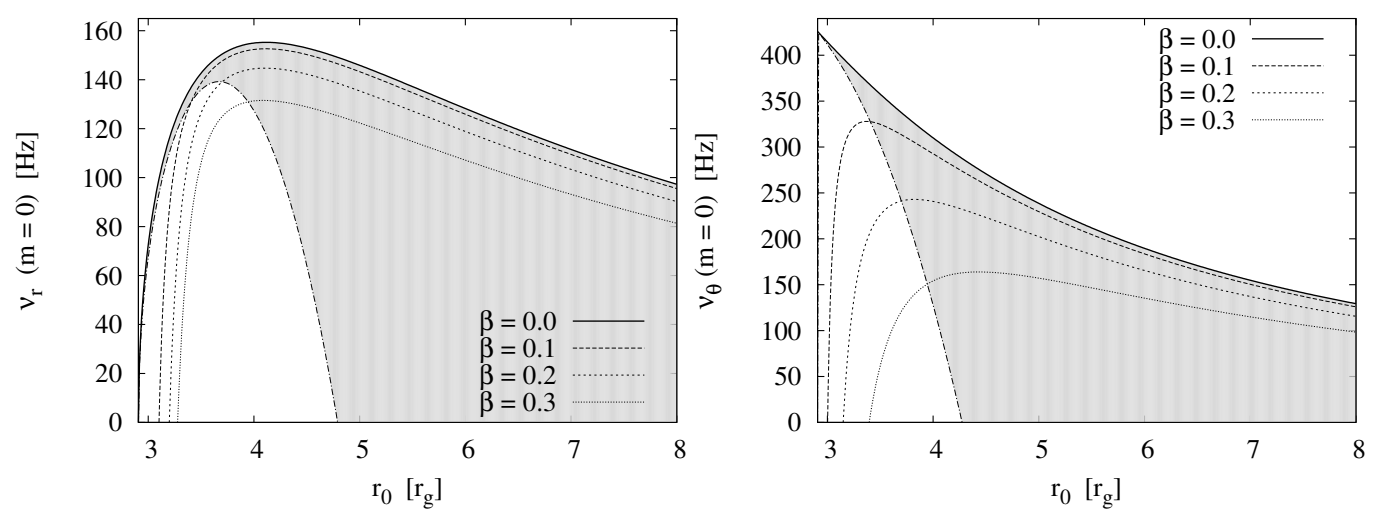

Figure 1: The axisymmetric epicyclic frequencies as functions of the torus pressure maximum $r_{0}$ for tori of various thickness $\beta$ that orbit a black hole of mass $M=10 \mathrm{M}_{\odot}$ and spin $a=0.8$. The allowed frequencies for equilibrium tori lie inside the shaded region. Left The radial epicyclic frequency. Right The vertical epicyclic frequency.

observed in the $\mathrm{kHz}$ range which corresponds to orbital frequencies just a few gravitational radii from the black hole. HF QPOs are thus another way to find the BH spin and come with some remarkable features (reviewed in [9]): They often appear in pairs of twin peaks which usually are in rational ratios, mostly in 3:2 ratio. Moreover, the very same QPO frequency pair reappears after years of a quiescent phase in the binary system - after the disc has been rebuilt completely. It is not clear where QPOs originate, but it seems that they are determined by the central compact object and that there is a resonance at play. As a matter of fact, the peculiar 3:2 ratio may be explained as a non-linear resonance between two (epicyclic) oscillations modes in the accretion flow [7].

\subsection{Epicyclic oscillations of non-slender tori}

The key to the nature of disc oscillations is that fluids in external gravity fields may oscillate with frequencies characteristic of the epicyclic motion of test particles: A small perturbation of a test particle orbiting on a geodesic line at $r=r_{0}$ (imagined as an infinitesimally slender torus) results in a radial and vertical epicyclic oscillation around the equilibrium point $r_{0}$ at a radial $\omega_{r 0}$ and vertical $\omega_{\theta 0}$ epicyclic frequency. Using a perturbation method one can derive fully general relativistic formulas for eigenfunctions and eigenfrequencies of the radial and vertical epicyclic modes of a slightly non-slender torus [11]. For an arbitrary BH spin one then finds that, in comparison with the epicyclic frequencies of free test particles, (axisymmetric) non-slender tori receive negative pressure corrections and exhibit thus lower frequencies (see Figure 1).

These two pressure corrected frequencies are now used to model the upper, $v_{U}$ and lower $v_{L}$ part of the QPO twin peaks.

$$
\frac{v_{U}}{v_{L}}=\frac{3}{2}, \quad v_{U}=\frac{\omega_{\theta}}{2 \pi}, \quad v_{L}=\frac{\Omega_{K}-\omega_{r}}{2 \pi} .
$$

The combination of frequency modes in (3.1) has been found by experience to fit best, because it is able to predict the whole range of BH spins $(|a| \leq 1)$. The choice is, however, somewhat ambiguous and it remains to be clarified which combination of frequency modes is ultimately involved in the QPO phenomenon. 


\section{Comparison}

In order to compare BH spin estimations for the three microquasar sources GRO J1655-40, XTE J1550-564 and GRS 1915+105 we calculate with (3.1) for every spin value $a$ and torus thickness $\beta$ the respective resonance radius, $r_{\text {res }}$ and resolve $M$ from $v_{U}\left(r_{r e s}\right)$. The best agreement so far is achieved with method 2 for rather thin accretion tori.

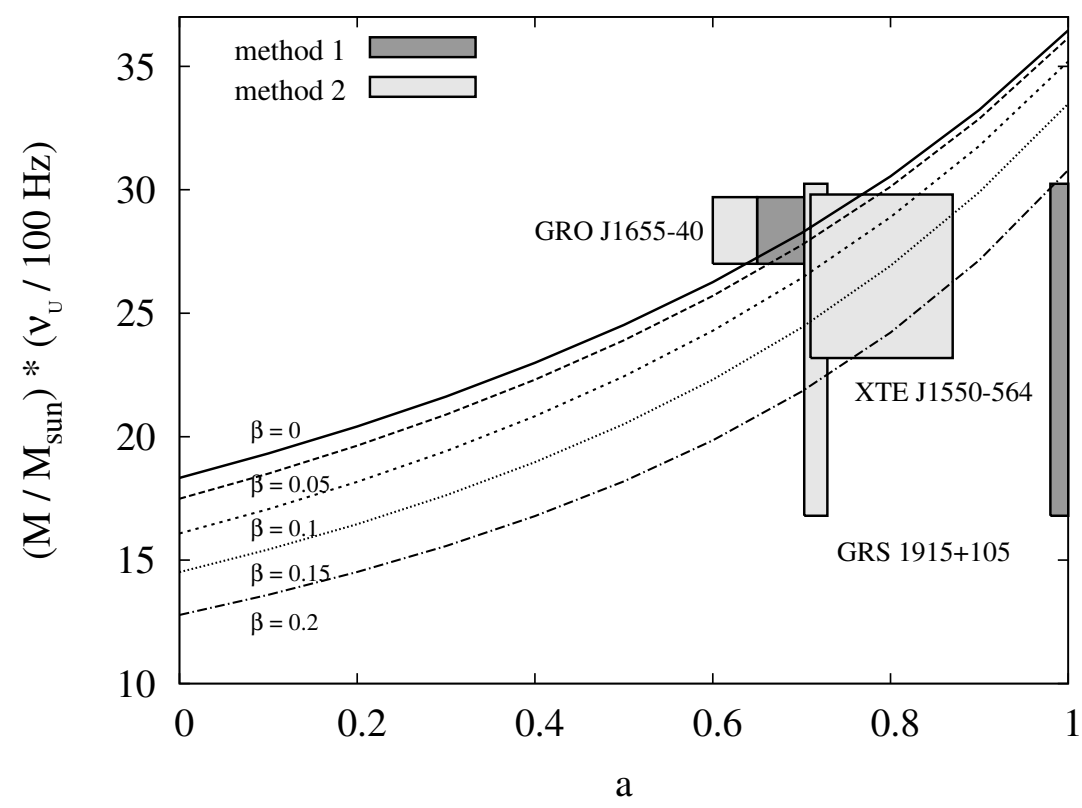

Figure 2: A preliminary comparison of different models to estimate the black hole spin parameter $a$. In shaded boxes, the spectral fitting method based on the standard thin disc model (method 1 for thermal spectra [8]; method 2 incl. comptonisation [6]). In solid and dashed lines, the QPO resonance method based on epicyclic oscillations of a non-slender fluid torus for different torus thickness $\beta$.

The main difficulty in estimating the spin of black holes using "spectral" methods like fitting of the observed spectra of microquasar states to these calculated is to model the complex physics of accreted matter. The QPO method is not influenced by details of the radiative transfer, lacks, however, still a unique way to determine the frequency mode pair. Nevertheless, it seems that both methods, reliable within their realm of applicability, are already able to give solid black hole spin estimates. And over the next months we anticipate performing deeper spin analysis with the improved slim disc spectral method as well as the QPO model.

\section{Acknowledgements}

It was a pleasure to be part of that fantastic workshop and I thank all that were involved in its organisation and realisation!

\section{References}

[1] Abramowicz M. A., Chen X.-M., Granath M., Lasota J.-P., 1996, ApJ, 471,762] 
[2] Blaes O. M., Arras P., Fragile P. C., 2006, MNRAS, 369, 1235

[3] Blaes O. M., Šrámková E., Abramowicz M. A., Kluźniak W., Torkelsson U., 2007, ApJ, 665642

[4] Davis S. W., Blaes O. M., Hubeny I., Tuner N. J., 2005, ApJ, 621, 372

[5] Davis S. W., Done C., Blaes O. M., 2006, ApJ, 647, 525

[6] Done C., Gierliki M., Kubota, A., 2007, A\&A Rev., 15, 1

[7] Kluźniak W., Abramowicz M. A., 2001, eprint arXiv:astro-ph/0105057

[8] McClintock J. E., Shafee R., Narayan R., Remillard R. A., Davis S. W., Li L., 2006, ApJ, 652, 518

[9] Remillard R. A., McClintock J. E., 2006, Ann. Rev. A\&A, 44, 49

[10] Shafee R., McClintock J. E., Narayan R., Davis S. W., Li L., Remillard R. A., 2006, ApJ, 636, 113

[11] Straub O., Šrámková E., 2009, CQG, 26, 055011 